\section{Effects of Primary Aortic Disease on Patient-Prosthetic Valve Mismatch}

To the Editor:

We read the article by Minakata et al with great interest. ${ }^{1}$ They presented their results of mechanical aortic valves in midlong-term follow-up. We have to admit that these results are astonishing. We believe the number of patients and the methodology are precise, but we also believe that the patient population is not homogeneous, considering some parameters such as previous aortic valve pathologies that the patients had. Because one should certainly clarify that aortic valve-related reoperations are mostly seen with prosthetic valve thrombosis, prosthetic valve dysfunction or patient size mismatch. Predictor factors for patient-valve mismatch vary in different studies. Some of the predictors are age, previous history of coronary intervention, hypertension, high body mass index, and aortic stenosis as a primary cause. ${ }^{2,3}$ Kaminishi et al claim that patients can have prosthetic valve mismatch especially with primary aortic stenosis due to annular calcifications and postinflammatory reactions. ${ }^{3}$ In this context, we believe that Minakata et al could have higher numbers of no aortic valve reoperation patients in their series if they only included patients with solidary aortic insufficiencies. We would be very happy to hear the authors' opinions on this matter.

\section{References}

1. Minakata K, Tanaka S, Okawa Y, Kaneko T, Okonogi S, Usui A, et al. Twenty-year outcome of aortic valve replacement with St. Jude Medical mechanical valves in Japanese patients. Circ J 2015; 79: $2380-2388$.

2. Pibarot P, Dumesnil JG. Hemodynamic and clinical impact of prosthesis-patient mismatch in the aortic valve position and its prevention. J Am Coll Cardiol 2000; 36: 1131-1141.

3. Kaminishi Y, Misawa Y, Kobayashi J, Konishi H, Miyata H, Motomura N, et al; Japan Cardiovascular Surgery Database Organization. Patient-prosthesis mismatch in patients with aortic valve replacement. Gen Thorac Cardiovasc Surg 2013; 61: 274-279.

Orhan Gokalp, MD

Bortecin Eygi, MD

Yuksel Besir, MD

Gamze Gokalp, MD

Ali Gurbuz, MD

Departmant of Cardiovascular Surgery, Faculty of Medicine (O.G., A.G.), Departmant of Cardiovascular Surgery, Ataturk Education and Research Hospital (B.E., Y.B.), Izmir Katip Celebi University, Izmir; Departmant of Pediatric Emergency, Tepercik Education and Research Hospital, Izmir (G.G.), Turkey

(Released online December 25, 2015) 